\title{
Uso inicial de tigeciclina en Argentina
}

\author{
Daniel Curcio, Francisco Fernández y Fernando Duret
}

\section{Initial use of tigecycline in Argentina}

Tigecycline is a new class of antibiotics active against emerging nosocomial pathogens as methicillinresistant Staphylococcus aureus, extended spectrum $\beta$-lactamases-producing enterobacteria and multidrugresistant Acinetobacter sp. It was approved for the treatment of complicated intra-abdominal infections and complicated skin and skin structure infections, but its pharmacological and microbiological profile is attractive to physicians for using it in other pathologies caused by resistant pathogens. The aim of this study was to evaluate in which indications tigecycline was used in Argentinian usual clinical settings during the first months after its launch. We analyzed tigecycline prescriptions in 69 patients with severe infections in 15 institutions. Fifteen patients (21\%) received tigecycline for approved indications, and 54 (79\%) for off label indications (56\% with scientific support and $23 \%$ with limited or without any scientific support). The most frequent off label use was ventilator associated pneumonia (VAP) (36 patients). The etiology of the infections was established in $61 \%$ of the patients. In all cases the isolated bacteria were multi-drug resistant (MDR) Acinetobacter $s p$.-carbapenems included. Our study shows that the off label use of tigecycline is frequent, specially in VAP. One of the reasons could be that for MDR-Acinetobacter sp. -VAP, there are few therapeutic options (for instance: colistin). Physicians must evaluate the benefits/risks to use this antibiotic for indications that lack rigorous scientific support.

Key words: Tigecycline, off label indications, ventilator associated pneumonia.

Palabras claves: Tigeciclina, indicaciones no aprobadas, neumonía asociada a ventilación mecánica.

\section{Introducción}

$\mathrm{T}$ igeciclina es el primero de una nueva clase de antimicrobianos denominados glicil-ciclinas. Es activo contra una gran variedad de microorganismos grampositivos y gramnegativos, incluyendo patógenos nosocomiales como Staphylococcus aureus meticilina-resistente (SAMR), enterobacterias productoras de $\beta$-lactamasas de espectro extendido (BLEEs) y Acinetobacter sp multi-resistente (MR) ${ }^{1}$.

Tigeciclina ha sido aprobada por la FDA (E.U.A), EMEA (Unión Europea) y la ANMAT (Argentina) para el tratamiento de infecciones intra-abdominales complicadas (IIAc) e infecciones de piel y tejido celular subcutáneo complicadas (IPTSCc), pero su perfil farmacológico y microbiológico resulta atractivo para emplearla además en otras patologías causadas por patógenos resistentes.

El uso no aprobado (off label) formalmente, se define como la prescripción de un fármaco para otras indicaciones que las aprobadas por las autoridades regulatorias. Las mismas pueden ser categorizadas como: con fuerte respaldo científico o con limitado o nulo respaldo científico.

El propósito de este estudio fue evaluar en qué indicaciones se prescribió tigeciclina en Argentina durante el primer mes posterior a su lanzamiento, y categorizar las prescripciones como aprobadas y no aprobadas, con o sin respaldo científico.

Secundariamente, registramos la gravedad de la enfermedad de los pacientes tratados y el éxito clínico obtenido con tigeciclina, según la evaluación del médico tratante.

\section{Pacientes y Métodos}

Tigeciclina (Tygacil $($ ) ) fue aprobada en Argentina en enero del 2006 y lanzada al mercado en abril del mismo año. Antes y después de su lanzamiento se iniciaron actividades educativas, llevadas a cabo por el fabricante, con el fin de difundir sus indicaciones ya aprobadas.

Durante el período julio-agosto del 2006 invitamos a un grupo médicos de 15 instituciones de Argentina, a completar una encuesta de datos mínimos relativos a sus pacientes tratados con este nuevo antimicrobiano, incluyendo: código del paciente, indicación para su uso, documentación microbiológica (si la hubiera), gravedad de la enfermedad, y existencia o no de resolución clínica de la infección (éxito o fracaso).

Las indicaciones fueron dividas en tres categorías:
Sanatorio San José, Buenos Aires, Argentina

Infectología Institucional SRL (DC, FF) Wyeth, Argentina (FD)

Recibido: 28 de agosto de 2006 Aceptado: 17 de mayo de 2007

Correspondencia a:

Daniel Curcio djcurcio@gmail.com djcurcio@intramed.net.ar 
Tabla 1. Tipo de indicación de tigeciclina, microbiología y éxito clínico

\begin{tabular}{|c|c|c|c|c|}
\hline Indicación & n (\%) & Diagnóstico (n) & $\begin{array}{c}\text { Documentación } \\
\text { microbiológica } 1 \%)\end{array}$ & $\begin{array}{c}\text { Éxito } \\
\text { clínico (\%) }\end{array}$ \\
\hline Tipo I & $15(21)$ & $\| A c^{2}(9) I P C S C C^{3}$ & 27 & 100 \\
\hline Tipo ॥ & $38(56)$ & NAVM $^{4}$ (36)NAC ${ }^{5}$ (2) & 58 & 92 \\
\hline \multirow[t]{6}{*}{ Tipo III } & $16(23)$ & $\operatorname{ITS}^{6}(8)$ & & \\
\hline & & mediastinitis (2) & & \\
\hline & & pie diabético (2) & 31 & 63 \\
\hline & & sepsis $\mathrm{OD}^{7}$ (3) & & \\
\hline & & osteomielitis (1) & & \\
\hline & & $\mathrm{IOP}^{8}(1)$ & & \\
\hline \multicolumn{5}{|c|}{$\begin{array}{l}{ }^{1} \text { en todos los casos Acinetobacter sp MR (incluidos carbepenems). }{ }^{2} \text { infecciones intra-abdomi- } \\
\text { nales complicadas. }{ }^{3} \text { infecciones de piel y celular subcutáneo complicadas. }{ }^{4} \text { neumonía asocia- } \\
\text { da a ventilación mecánica (en un caso con IIAc). }{ }^{5} \text { neumonía adquirida en la comunidad. } \\
{ }^{6} \text { infecciones del torrente sanguíneo. }{ }^{7} \text { sepsis de origen desconocido. }{ }^{8} \text { infección ortopédica- } \\
\text { protésica }\end{array}$} \\
\hline
\end{tabular}

tipo I-aquellas indicaciones aprobadas, tipo II-aquellas indicaciones no aprobadas, pero con determinado respaldo científico (perfil microbiológico y farmacodinámico del fármaco y existencia de ensayos clínicos en marcha) y tipo III-aquellas indicaciones no aprobadas y con escaso respaldo científico.

Análisis estadístico. Las proporciones se expresaron como porcentaje con $95 \%$ de intervalo de confianza corregido (ICC).

\section{Resultados}

Fueron analizadas 69 prescripciones de tigeciclina, voluntariamente enviadas por el médico tratante entre julio 15 y agosto 31 del 2006. Quince pacientes (21\% ICC 11,3-32,2\%) recibieron tigeciclina por indicaciones tipo I. En 79\% de los casos (54/69 de los pacientes ICC $73,2-93 \%)$ la prescripción de tigeciclina fue del tipo no aprobada (tipo II $56 \%$-38/69- y tipo III $23 \%$ - 16/69-). El uso no aprobado más frecuente fue la neumonía asociada a ventilación mecánica (NAVM (95\% ICC 86,3$100 \%$ de las indicaciones tipo II) (Tabla 1).

Según los médicos tratantes, 57 pacientes ( $88 \%$ ICC 79-96,4\%) tuvieron infecciones graves: $61,5 \%$ objetivada a partir de la presencia de falla multi-orgánica, $35 \%$ medida por el puntaje APACHE (Acute Physiology, Age, Chronic Health Evaluation) II y 3,5\% medida por el puntaje SOFA (Sequential Organ Failure Assessment).

El éxito clínico se obtuvo en 56 pacientes (86\% -ICC
$77-95,3 \%$-); en 100, 92 y $63 \%$ de las indicaciones tipo I, II y III, respectivamente. Falla de tratamiento se observó en tres pacientes con indicaciones del tipo II (todos con NAVM, en un caso combinada con una IIAc) y en seis pacientes con indicaciones del tipo III (dos infecciones de pie en pacientes diabéticos, dos infecciones ortopédico-protésicas y dos infecciones del torrente sanguíneo ITS-ITS).

La etiología de las infecciones fue establecida en 32 pacientes (50\% IC 36,3-62,2\%), En todos los casos, la bacteria aislada fue Acinetobacter sp. multi-resistentes (MR).incluidos los carbapenems: indicaciones $27 \%$ $(4 / 15), 58 \%(22 / 38)$ y $31 \%(5 / 16)$ del tipo I, II y III, respectivamente.

\section{Discusión}

La prescripción de fármacos para indicaciones no aprobadas es amplia en el tratamiento del SIDA, cáncer y afecciones pediátricas, pero no se limita sólo a estas áreas ${ }^{2}$.

Nuestro estudio muestra que el uso no aprobado del nuevo antimicrobiano, tigeciclina, fue de $79 \%$. La NAVM representó el primer diagnóstico para el uso no aprobado (67\%), evidenciándose en estos casos una eficacia clínica de 92\% (33/36 patients). Las indicaciones aprobadas (tipo I) tuvieron 100\% de éxito clínico, en oposición con las indicaciones tipo III cuyo éxito alcanzó a 57\%. Todas las infecciones microbiológicamente documentadas fueron causadas por Acinetobacter sp. MR

De acuerdo con los resultados del Proyecto VALIDAR, en Argentina, la NAVM es la infección nosocomial más frecuente y Acinetobacter sp es uno de los microorganismos predominantes $(30 \%$ de las infecciones microbiológicamente documentadas) $)^{3}$. Esta información es similar a los datos del Programa SIR (Sistema Informático de Resistencia), que mostró $60 \%$ de resistencia en Acinetobacter sp en las unidades de cuidados intensivos argentinos ${ }^{4}$.

En relación con infecciones graves (NAVM, ITS, IIAc), la terapia antimicrobiana inadecuada se asoció con peor pronóstico, en términos de resolución clíni$\mathrm{ca}^{5-7}$. En una encuesta efectuada a 87 médicos intensivistas, $91 \%$ de ellos consideró un tratamiento apropiado si el antimicrobiano era activo contra el patógeno y si el perfil farmacológico era adecuado para el sitio de la infección, sin considerar las indicaciones aprobadas (Curcio D. datos no publicados). Las razones de estos conceptos podrían ser: los médicos tienen otras opciones terapéuticas efectivas para las indicaciones aprobadas (cIAI, piel TCSCc); tigeciclina es activa contra los patógenos nosocomiales más fre- 
cuentes -excepto Pseudomonas aeruginosa- y para la NAVM causada por Acinetobacter sp hay muy pocas opciones terapéuticas. Por ejemplo, colistín, un antimicrobiano frecuentemente usado en estos pacientes, tiene limitada evidencia acerca de su eficacia clínica y potencial toxicidad, sobre todo sobre el riñón.

En el caso particular de tigeciclina, estamos a la espera de las resultados finales de varios ensayos clínicos en marcha para indicaciones diferentes que IIAc e IPTSCc como NAVM, neumonía adquirida en la comunidad e infecciones causadas por patógenos resistentes. Conocer estos resultados es fundamental para apoyar su uso empírico inicial en estas indicaciones.

\section{Resumen}

Tigeciclina es una nueva clase de antimicrobiano activo contra patógenos nosocomiales emergentes como Staphylococcus aureus resistente a meticilina, enterobacterias productoras de $\beta$-lactamasas de espectro extendido y Acinetobacter sp multi-resistente. Hasta el momento, ha sido aprobado para el tratamiento de infecciones intra-abdominales complicadas e infecciones de piel y tejido celular subcutáneo complicadas, pero su perfil farmacológico y microbiológico re- sulta atractivo para prescribirlo en otras patologías, sobre todo aquellas causadas por patógenos resistentes. El objetivo de este estudio fue evaluar en qué indicaciones fue utilizada tigeciclina en Argentina durante el primer mes después de su lanzamiento. Para tal fin, fueron analizadas las prescripciones de tigeciclina en 69 pacientes con infecciones graves en 15 instituciones. Quince pacientes (21\%) recibieron tigeciclina para indicaciones aprobadas y $54(79 \%)$ para indicaciones no aprobadas - off label- $(56 \%$ con respaldo científico y $23 \%$ con mínimo o nulo respaldo científico). La indicación más frecuente, dentro de las no aprobadas, fue la neumonía asociada a ventilación mecánica (NAVM) (36 pacientes). Se demostró la etiología de las infecciones en $61 \%$ de los pacientes. En todos ellos las bacterias aisladas fueron Acinetobacter sp multi-resistente (MR) incluidos los carbapenemes. Nuestro estudio muestra que la prescripción de tigeciclina para indicaciones no aprobadas es frecuente, especialmente en NAVM. Dentro de las razones que podrían explicar este fenómeno, se encuentra el escaso número de opciones terapéuticas para el tratamiento de infecciones graves por Acinetobacter sp (p. ej: colistín). Sin embargo, es aconsejable que los médicos evalúen el beneficio/riesgo de usar este antimicrobiano para indicaciones que carecen aún de respaldo científico consistente.

\section{Referencias}

1 - Frampton J E, Curran M P. Tigecycline. Drugs 2005; 65: 2623-35

2- Kos M, Wertheimer A I, Mrhar A. Satisfaction with pharmacotherapy for approved and off -label indications- a Delphi study. Ann Pharmacother 2005; 39: 649-54.

3- Quirós R. Proyecto VALIDAR. Evaluación de los resultados, implicancias para nuestro país. Workshop. VI Congreso Argentino de Infectología. 2006.

4- Quinteros M, Radice M, Famiglietti A,
Marín M, Nicola F, Casellas J M, et al. Análisis de la sensibilidad a los antimicrobianos en los aislamientos de pacientes internados. Años 2004-2005. Sistema Informático de Resistencia (SIR) (SADEBAC-AAM). Boletín de la Asociación Argentina de Microbiología 2006; 172: 13-6.

5- Kang C I, Kim S H, Park W B, Lee K D, Kim H B, Kim E C, et al. Bloodstream infections caused by antibiotic-resistant gram-negative bacilli: risk factors for mortality and impact of inappropriate initial antimicrobial therapy on outcome.
Antimicrob Agents Chemother 2005; 49: 760-6.

6- Bare M, Castells X, García A, Riu M, Comas M, Egea M J. Importance of appropriateness of empiric antibiotic therapy on clinical outcomes in intraabdominal infections. Int $\mathrm{J}$ Technol Assess Health Care 2006; 22: 242-8.

7- Luna C, Aruj P, Niederman M, Garzón J, Violi D, Prignoni A, et al Appropriateness and delay to initiate therapy in ventilatorassociated pneumonia. Eur Respir J 2006; 27: $158-64$. 